\title{
FLUTUAÇÃO POPULACIONAL E IMPACTO DE ARTRÓPODES \\ PREDADORES SOBRE Leptopharsa heveae DRAKE \& POOR (HETEROPTERA: TINGIDAE) EM SERINGUEIRA
}

Fernando da Silva Fonseca ${ }^{1}$

Francisco Jorge Cividanes ${ }^{2}$

Terezinha Monteiro dos Santos ${ }^{3}$

Júlio Cesar Galli ${ }^{2}$

\section{RESUMO}

Determinou-se a flutuação populacional e o potencial de impacto de artrópodes predadores sobre o percevejo-de-renda, Leptopharsa heveae Drake \& Poor, em seringueira, Hevea brasiliensis Müell Arg. O levantamento foi conduzido em seringueiras do clone PB 235, durante outubro de 1998 a novembro de 1999, em Pindorama, SP. A amostragem dos artrópodes predadores foi obtida coletando-se folhas das partes interna e externa dos terços inferior, médio e superior dos lados norte e sul das seringueiras. $O$ impacto da predação foi verificado por meio de correlação linear simples. Os artrópodes predadores que predominaram no levantamento foram: Araneae (Anyphaenidae, Araneidae, Dictynidae e Salticidae); Coccinellidae [Cycloneda sanguinea Linnaeus, Diomus sp. e Scymnus (Scymnus) sp.] e Chrysopidae. As aranhas foram mais numerosas de março a junho, o mesmo ocorrendo com os coccinelídeos de maio a

${ }^{1}$ Plantações E. Michelin Ltda., Rodovia BR-163, Km 16,5. Caixa Postal 80, 78700090, Rondonópolis, MT.

${ }^{2}$ Depto. Fitossanidade, FCAV/UNESP, Via de Acesso Prof, Paulo D. Castellane s/n, 14884-900, Jaboticabal, SP.

${ }^{3}$ Depto. Entomologia, Universidade Federal de Lavras, Caixa Postal 37 37200-000, Lavras, MG. 
novembro e com os crisopídeos de março a novembro. Apenas as aranhas apresentaram potencial de impacto sobre $L$. heveae.

Palavras-chave: Controle biológico, percevejo-de-renda, aranhas, Hevea brasiliensis.

\title{
POPULATION FLUCTUATION AND IMPACT OF ARTHROPOD PREDATORS ON Leptopharsa heveae DRAKE \& POOR (HETEROPTERA: TINGIDAE) IN RUBBER TREE
}

\begin{abstract}
A populational survey of arthropod predators was carried out aiming to determine their potential impact on the rubber tree lacebug, Leptopharsa heveae Drake \& Poor, in rubber tree, Hevea brasiliensis Müell Arg. In Pindorama State of São Paulo, Brazil. The survey was done by sampling north and south sides of rubber trees clone PB 235, considering leaves of internal and external parts of the top, middle and basal sections of the trees. The impact of arthropod predators was verified using simple linear correlation. Araneae (Anyphaenidae, Dictynidae, Salticidae and Araneidae); Coccinellidae [Cycloneda sanguinea, Diomus sp., Scymnus (Scymnus) sp.] and Chrysopidae were the predators that prevailed in the survey. Spiders were more numerous from March to June, the same being observed with coccinellids from May to November and chrysopids from March to November. Only spiders showed potential impact on $L$. heveae.
\end{abstract}

Key words: Biological control, lacebug, spiders, Hevea brasiliensis.

\section{INTRODUÇÃO}

O percevejo-de-renda, Leptopharsa heveae Drake \& Poor (Hemiptera: Tingidae), encontra-se entre os principais insetos praga da seringueira, Hevea brasiliensis Müell Arg. Esse inseto, ao sugar a seiva das 
folhas, diminui a capacidade fotossintética da planta reduzindo a produção de látex em até 30\% (ABREU, 1996). O controle de $L$. heveae pode ser obtido aplicando-se o fungo Sporotrix insectorum Hoog \& Evans (SOSAGOMEZ \& LANTERI, 1999; JUNQUEIRA et al., 1999). A eficiência desse agente de controle biológico está relacionada diretamente às condições ambientais e à densidade populacional da praga, podendo atingir $94 \% \mathrm{em}$ épocas úmidas, mas apenas $26 \%$ em períodos secos, quando se faz necessário o emprego de outros inimigos naturais para que seja obtido sucesso no controle de $L$. heveae (COSTA et al., 2003). No Brasil, os registros sobre predadores associados a insetos-praga da seringueira referem-se às famílias Carabidae, Chrysopidae, Coccinellidae, Forficulidae, Labiduridae, Reduviidae e Syrphidae (BERGMANN et al., 1991; FAZOLIN, 1991; SCOMPARIN, 1997; TANZINI, 1997). No estado de São Paulo, RINALDI \& RUIZ (2002) caracterizaram a araneofauna em seringais relatando a ocorrência de elevada densidade de aranhas nas copas das plantas, enquanto COSTA et al. (2003) constataram o parasitismo de ovos de L. heveae por Erythmelus tingitiphagus (Soares) (Hymenoptera: Mymaridae). Na Colômbia, formigas do gênero Crematogaster foram indicadas como predadores importantes do tingídeo Leptopharsa gibbicarina Froeschner em seringueira (MONTANEZ et al., 1998; SALAMANCA et al., 2000). Os artrópodes predadores desempenham expressivo papel como controladores da densidade populacional de insetos herbívoros (WILSON et al., 1991). Uma indicação preliminar do impacto desses organismos sobre pragas pode ser obtido correlacionando-se o número do predador e da presa encontrados nas diferentes amostras do levantamento populacional. As correlações positivas indicam certo grau de especificidade do predador pela presa, podendo ser esperado uma resposta numérica rápida à variação da densidade da presa. A correlação positiva também ocorre quando o predador apresenta baixa taxa de ataque ou 
quando o crescimento populacional da presa for pouco significativo. Por outro lado, as correlações negativas indicam resposta numérica lenta ou atrasada do predador com relação à mudança da densidade da presa. Tais respostas são encontradas em predadores altamente polífagos que podem iniciar o ataque a determinada presa apenas quando essa apresentar densidade relativamente elevada. As correlações negativas ocorrem também com presas que tendem mostrar rápida mudança de abundância ou com predadores com taxa de ataque elevada (KIDD \& JERVIS, 1996).

No presente trabalho, realizou-se um levantamento populacional de artrópodes predadores associados à seringueira objetivando-se determinar a flutuação populacional e o potencial de impacto desses inimigos naturais sobre L. heveae.

\section{MATERIAL E MÉTODOS}

O estudo foi realizado na Estação Experimental do Instituto Agronômico de Campinas (IAC), no município de Pindorama, SP (altitude $594 \mathrm{~m} ; 21^{\circ} 13^{\prime}$ de latitude Sul e $48^{\circ} 55^{\prime}$ de longitude Oeste).

O levantamento populacional de $L$. heveae e artrópodes predadores foi realizado em seringal do clone PB 235, com árvores de aproximadamente $7 \mathrm{~m}$ de altura. As amostragens foram efetuadas, em média, a cada 19 dias, durante o período de outubro/1998 a novembro/1999, totalizando 22 épocas de amostragens. Em cada época de amostragem, 10 árvores foram escolhidas ao acaso amostrando-se os lados voltados para o norte e sul, nos terços inferior ( 1 a $3 \mathrm{~m}$ ), médio ( 3 a $5 \mathrm{~m}$ ) e superior ( 5 a 7 m) conforme metodologia de BATZER et al. (1995). Em cada terço, cinco folhas da metade interna e cinco folhas da metade externa dos ramos foram escolhidas ao acaso e retiradas, coletando-se 600 folhas por época de amostragem. Cada grupo de cinco folhas foi mantido em saco plástico e a 
avaliação do número de artrópodes predadores e de ninfas e adultos de $L$. heveae foi efetuada em laboratório.

A coleta das folhas localizadas no terço inferior e médio das seringueiras foi efetuada utilizando-se escada com alcance de $5,40 \mathrm{~m}$. As folhas localizadas no terço superior foram amostradas empregando-se escada e um gancho com cabo de $2 \mathrm{~m}$ de comprimento, que possibilitou puxar os ramos para baixo e coletar as folhas. O impacto dos predadores sobre $L$. heveae foi verificado por meio de correlação linear simples. As correlações foram processadas entre o número médio de ninfas e adultos de L. heveae amostrados nas diferentes partes das plantas e o número total de indivíduos pertencentes às espécies ou famílias de artrópodes predadores.

\section{RESULTADOS E DISCUSSÃO}

Os artrópodes predadores que predominaram durante 0 levantamento populacional foram aranhas, coccinelídeos e crisopídeos. Espécimens desses grupos ocorreram durante todo o período de amostragem, exceto coccinelídeos em 30/10/98 e 19/04/99 (Figura 1). As aranhas foram mais abundantes, representando $51,1 \%$ do total capturado, seguidas por crisopídeos $(27,1 \%)$ e coccinelídeos $(15,8 \%)$. Insetos predadores das famílias Hemerobiidae, Mantispidae e Formicidae ocorreram esporadicamente e representaram apenas $6 \%$ do total capturado. Destaca-se que a presença de insetos predadores de determinado grupo em uma cultura específica pode estar relacionada com as características da planta hospedeira (RIUDAVETS \& CASTANÉ, 1998). Com relação aos coccinelídeos, a preferência desses predadores pelo hábitat é determinada principalmente pelas condições microclimáticas, existindo espécies que ocorrem em apenas um tipo de cobertura vegetal, isto é, árvores, arbustos ou plantas cultivadas (IPERTI, 1999). 
As aranhas encontradas no levantamento pertencem às famílias Anyphaenidae, Araneidae, Dictynidae, Heteropodidae, Mimetidae Salticidae, Theridiidae, Thomisidae e Titanoecidae. Entre os coccinelídeos as seguintes espécies foram encontradas: Azya luteipes Mulsant, Cycloneda saguinea Linnaeus, Diomus sp., Pentilia sp., Psyllobora confluens Fabricius, Scymnus (Scymmus) sp. e Stethorus sp.

O maior número de aranhas foi observado de meados de março até final de junho de 1999, apresentando vários picos populacionais com o maior deles ocorrendo em 04/06/1999 (Figura 1). Espécimens das famílias Anyphaenidae, Araneidae, Dictynidade e Salticidae prevaleceram durante o período estudado, sendo capturados em todas as datas de amostragem. Esses resultados concordam parcialmente com os de RINALDI \& RUIZ (2002), que indicaram Anyphaenidae, Salticidae e Theridiidae como as famílias de aranhas mais abundantes em seringais paulistas.

Os coccinelídeos tornaram-se mais numerosos a partir de meados de maio até meados de novembro de 1999, com picos populacionais em 20/05 e 24/08/1999 (Figura 1), enquanto os crisopídeos ocorreram em maior número do início de março até meados de novembro de 1999, apresentando pico populacional em 04/05/1999. As espécies de coccinelídeos C. sanguinea, Diomus sp. e Scymnus (Scymnus) sp. foram mais abundantes que as demais, ocorrendo em $91 \%$ das amostras do levantamento. FAZOLIN (1991) constatou a presença de C. sanguinea em seringueiras no município de Rio Branco, AC, enquanto BERGMANN et al. (1994) associaram C. sanguinea e Scymnus sp. à seringueiras na região de Bálsamo, SP.

Os coeficientes de correlação foram significativos apenas entre a variação da densidade populacional de adultos de $L$. heveae e de aranhas da família Dictynidae $(r=0,54)$ e do total de aranhas capturadas $(r=0,61$, Tabela 1). $O$ fato das ninfas de tingídeos apresentarem estruturas morfológicas relacionadas ao comportamento defensivo (MASON, 1991) 
por secretarem substâncias repelentes e feromônios de alarme (ALDRICH et al., 1991) que as possibilitam evitar o ataque de predadores (GUILBERT, 2004) pode ser uma provável explicação para a não obtenção de correlação significativa entre as ninfas de $L$. heveae e os predadores. Segundo KIDD \& JERVIS (1996), correlações positivas e significativas podem indicar as seguintes características de artrópodes predadores e presas: 1) especificidade da presa por parte do predador, que apresenta rápida resposta numérica às variações de densidade da presa e 2) presa com baixa taxa de crescimento populacional. De acordo com DALL'OGLIO et al. (1999), L. heveae possui elevada taxa de crescimento populacional. Assim, o valor positivo e significativo dos coeficientes de correlação obtidos para aranhas no presente estudo (Tabela 1) sugerem que esses predadores podem ter apresentado especificidade com relação à presa e expressivo aumento populacional em resposta ao crescimento da população de adultos de $L$. heveae, com destaque para a família Dictynidae. De fato, pela Figura 1 verifica-se que a densidade populacional das aranhas aumentou com o crescimento populacional do tingídeo, com períodos de densidade desses predadores coincidindo com aqueles de maior número das ninfas e adultos de $L$. heveae em 30/03/99 e com adultos do tingídeo em 04/06/99. As ninfas e principalmente os adultos aumentaram de densidade a partir de meados de setembro de 1999, o mesmo ocorrendo com as aranhas. Constatou-se também que os menores níveis populacionais das aranhas ocorreram quando foram mínimas as densidades de ninfas e adultos de $L$. heveae. Vários autores (TAVELLA \& ARZONE, 1987; TRUMBULE et al., 1995; SATHIAMMA et al., 1998; UNIMON \& RANJITH, 1998) indicaram aranhas como inimigos naturais de tingídeos. Deve ser ressaltado que tais predadores ocorrem em abundância nas culturas apresentando elevado potencial de controle natural de populações de pragas (RINALDI, 1995; RINALDI \& RUIZ, 2002). 
Os coeficientes de correlação não significativos obtidos entre $L$. heveae e coccinelídeos e crisopídeos (Tabela 1) sugerem que esses predadores tiveram pouca importância no controle natural do percevejo-derenda. Apesar de alguns autores (SCHOOVEN et al., 1975; BATISTA FILHO et al., 1995) terem indicado esses grupos como inimigos naturais de tingídeos, inclusive de $L$. heveae, as correlações não significativas obtidas no presente estudo podem estar relacionadas com a preferência desses predadores por determinadas espécies de presas. Os coccinelídeos e crisopídeos são reconhecidamente polífagos, entretanto, muitos desses predadores são preferencialmente afidófagos, principalmente as joaninhas C. sanguinea e Scymnus (Scymmus) sp. (VANDENBERG \& GORDON, 1988; NARANJO et al., 1990; IPERTI, 1999; STELZL \& DEVETAK, 1999).

De modo geral, os períodos de maior densidade dos coccinelídeos não coincidiram com os de maior número de $L$. heveae (Figura 1). As populações de ninfas e adultos do percevejo decresceram durante maio até final de agosto, ocorrendo praticamente o oposto com os coccinelídeos. Durante o período de aumento populacional de $L$. heveae, iniciado em meados de setembro de 1999, esses predadores tenderam a decrescer em número, o mesmo acontecendo com os crisopídeos a partir do final de setembro. O fato da densidade populacional de adultos do percevejo-derenda ter aumentado e permanecido elevada durante o período de incremento e pico populacional dos crisopídeos, observado de março a maio de 1999, pode ser considerado mais uma evidência da inexistência de pressão predatória sobre $L$. heveae.

\section{CONCLUSÕES}

1. Os predadores de ocorrência mais comum em seringueira são aranhas das famílias Anyphaenidae, Dictynidae, Salticidae e Araneidae; 
coleópteros da família Coccinellidae, das espécies C. saguinea, Diomus sp. e Scymnus (Scymnus) sp. e neurópteros da família Chrysopidae.

2. As aranhas apresentam maior densidade populacional de março a junho, o mesmo ocorrendo com os coccinelídeos de maio a novembro e com os crisopídeos de março a novembro.

3. Espécimens do grupo Araneae, principalmente a família Dictynidae, são os artrópodes predadores com maior potencial de impacto sobre $L$. heveae.

\section{AGRADECIMENTOS}

À FAPESP, pelo financiamento da pesquisa (processo 98/106575); ao Dr. Richard C. Froeschner, Smithsonian Institution, Washington, D.C., E.U.A., pela identificação de L. heveae; à Dra Isabela M. P. Rinaldi, Instituto de Biociências/UNESP, Botucatu, SP, pela identificação de Araneae; à $\mathrm{Dr}^{\mathrm{a}}$. Juliane Milléo, Centro de Identificação de Insetos Fitófagos/UFPR, Curitiba, PR, pela identificação de Coccinellidae e ao $\mathrm{CNPq}$, pela bolsa concedida ao segundo autor.

\section{REFERÊNCIAS BIBLIOGRÁFICAS}

ABREU, J. M. de, 1996. Aspectos bioecológicos e controle das principais pragas da seringueira no Brasil. Ilhéus, CEPLAC/CEPEC. 20p.

ALDRICH, J. R.; J. W. NEAL; J. E. OLIVER \& W. R. LUSBY, 1991. Chemistry vis-à-vis maternalism in lace bugs (Heteroptera: Tingidae): alarm pheromones and exudate defense in Corythucha and Gargaphia species. Journal of Chemical Ecology, 17: 2307-2322.

BATISTA FILHO, A.; L. G. LEITE \& A. P. SILVEIRA, 1995. Ocorrência da mosca-de-renda, Leptopharsa heveae, em Buritama, SP. Arquivos do Instituto Biológico, 62: .81. 
BATZER, H. O.; M. P. MARTIN; W. J. MATTSON \& W. E. MILLER, 1995. The forest tent caterpillar in aspen stands: distribution and density estimation of four life stages in four vegetation strata. Forest Science, 41 (1): 99-121.

BERGMANN, E. C.; S. D. L. IMENES, \& M. T. TAVARES, 1991. Ocorrência da cochonilha Aspidiotus sp. (Homoptera-Diaspididae) e seus parasitóides em clones de seringueira, no estado de São Paulo. Arquivos do Instituto Biológico, 58 (1/2): 65-67.

BERGMANN, E. C.; S. L. IMENES; A. M. TEMPEST; L. E. F. R. SILVA; C. CAMPANER \& M. F. STRADIOTO, 1994. Contribuiçâo ao conhecimento da entomofauna em cultura de seringueira (Hevea brasiliensis). Cultura Agrônomica, 3 (1): 33-46.

COSTA, V. A.; C. F. PEREIRA \& A. BATISTA FILHO, 2003. Observações preliminares sobre o parasitismo de ovos de Leptopharsa heveae (Hemiptera: Tingidae) em seringueira em Pindorama, SP. Arquivos do Instituto Biológico, 70 (1): 225-226.

DALL'ÓGLIO, O. T.; O. PERES FILHO \& M. J. DUDA, 1999. Flutuação populacional de Leptopharsa heveae Drake \& Poor (Hemiptera: Tingidae) em seringueira no Estado de Mato Grosso. In: CONGRESSO BRASILEIRO DE ENTOMOLOGIA, 17. Rio de Janeiro. Resumos. v. 2, p. 732.

FAZOLIN, M., 1991. Análise faunística de insetos coletados com armadilhas em seringueira no Acre. Piracicaba. 236p. (Doutorado ESALQ/USP).

GUILBERT, E., 2004. Do larvae envolve the same way as adults in Tingidae (Insecta: Heteroptera)? Cladistics, 20: 139-150.

IPERTI, G., 1999. Biodiversity of predaceous coccinellidae in relation to bioindication and economic importance. Agriculture, Ecosystems and Environment, 74 (1-3): 323-342. 
JUNQUEIRA, N. T. V; E. PINHEIRO; R. T. ALVES; P. CELESTINO FILHO; A. V. PEREIRA; M. A. S. OLIVEIRA; J. F. FIALHO \& L. GASPAROTTO, 1999. Controle biológico do percevejo-de-renda (Leptopharsa heveae Drake \& Poor) em seringais de cultivo. Circular Técnica EMBRAPA Cerrados, n.3,30p.

KIDD, N. A. C. \& JERVIS, M. A. 1996. Population dynamics, p. 293-374. In: M. JERVIS \& N. KIDD (Eds.) Insect natural enemies, practical approaches to their study and evaluation. London: Chapman \& Hall.

MASON, J. R.; J. NEAL; J. E. OLIVER \& W. R. LUSBY, 1991. Birdrepellent properties of secretions from nymphs of the Azalea lace bug. Ecological Applied, 1: 226-230.

MONTANEZ, M. L.; H. C. GUERRERO; J. E. LUQUEZ \& A. MENDEZ, 1998. Control biologico de Leptopharsa gibbicarina (Hemiptera: Tingidae) com la hormiga Crematogaster sp. (Hymenoptera: Formicidae) en palma de aceite. Revista Colombiana de Entomologia, 24 (3/4): 89-94.

NARANJO, S. E.; R. L. GIBSON \& D. D. WALGENBACH, 1990. Developmental, survival, and reproduction of Scymnus frontalis (Coleoptera: Coccinellidae), an imported predator of Russian wheat aphid, at four fluctuating temperatures. Annals of the Entomological Society of America, 83 (3): 527-531.

RINALDI, I. M. P., 1995. Aranhas no controle biológico de insetos: fatos e perspectivas. In: CICLO DE PALESTRAS SOBRE CONTROLE BIOLÓGICO DE PRAGAS, 5, Campinas. Anais. p. 155-177.

RINALDI, I. M. P. \& G. R. S. RUIZ, 2002. Comunidades de aranhas (Araneae) em cultivos de seringueira (Hevea brasiliensis Muell. Arg.) no estado de São Paulo. Revista Brasileira de Zoologia, 19 (3): 781 788. 
RIUDAVETS, J. \& C. CASTANÉ, 1998. Identification and evaluation of native predators of Frankliniella occidentalis (Thysanoptera: Thripidae) in the mediterranean. Environmental Entomology, 27 (1): 86-93.

SALAMANCA, O. J. C.; G. H. CALVACHE; J. A. TORRE; N.C. MESA \& A. MENDEZ, 2000. Aspectos ecologicos de Crematogaster spp. (Hymenoptera: Formicidae) depredador de Leptopharsa gibbicarina (Hemiptera: Tingidae) en palma de aceite Elaeis guineensis. Revista Colombiana de Entomologia, 26 (1/2): 61-66.

SATHIAMMA, B.; K. R. C. NAIR \& V. P. SONIYA, 1998. Additional record of insect predators of coconut lace bug Stephanitis typica (Distant) and studies on biology and feeding potential of Euagoras plagiatus Burmeister (Heteroptera: Reduviidae). Entomon, 24 (2): 165-171.

SCOMPARIN, C. H. J., 1997. Crisopídeos (Neuroptera: Chrysopidae) em seringueira (Hevea brasiliensis Müell. Arg.) e seu potencial no controle biológico de percevejo-de-renda (Leptopharsa heveae Drake \& Poor) (Hemiptera: Tingidae). Jaboticabal. 147p. (Mestrado - FCAV/UNESP).

SCHOONHOVEN, A. van; F. BURBANO \& R. ARENAS, 1975. Notes on the biology of the lace bug Gargaphia sanchezi (Hemiptera: Tingidae) a pest of beans (Phaseolus vulgaris). Turrialba, 25 (3): 327. Disponível em: <http://www.gateway.ovid.br>. Acesso em: 18 abr. 2005.

SOSA GOMEZ, D. R. \& A. A. LANTERI, 1999. Estado actual del control biologico de plagas agricolas com hongos entomopatogenos. Revista de la Sociedad Entomologica Argentina, 58 (1/2): 295-300.

TAVELLA, L. \& A. ARZONE, 1987. Indagini sui limitatori di Corythucha ciliata (Say) (Rhynchota: Heteroptera). Redia, 70: 443-457. 
STELZL, M. \& D. DEVETAK, 1999. Neuroptera in agricultural ecosystems. Agriculture, Ecosystems and Environment, 74: 305321.

TANZINI, M. R., 1997. Controle biológico do percevejo-de-renda da seringueira. In: CICLO DE PALESTRAS SOBRE CONTROLE BIOLÓGICO DE PRAGAS, 5., Campinas. Anais. p. 32-38.

TRUMBULE, R. B.; R. F. DENNO \& M. J. RAUPP, 1995. Management considerations for the azalea lace bug in landscape habitats. Journal of Arboriculture, 21 (2): 63-68. Disponível em: $<$ http://www.gateway.ovid.br>. Acesso em: 18 abr. 2005.

UNIMON, N. K. \& A. M. RANJITH, 1998. Natural enemy complex of coconut lacewing bug Stephanitis typicus Distant and predatory potential of major natural enemies. Journal of Tropical Agriculture, $36(1 / 2): 81-82$.

VANDENBERG, N. \& R. D. GORDON,.1988. The Coccinellidae (Coleoptera) of South America, part I. A revision of the genus Erythroneda Timberlake, 1943. Revista Brasileira de Entomologia, 32: 31-43.

WILSON, L. T.; P. J. TRICHILO \& D. GONZALEZ, 1991. Natural enemies of spider mites (Acari: Tetranychidae) on cotton: density regulation or casual association? Environmental Entomology, 20 (3): 849-856. 

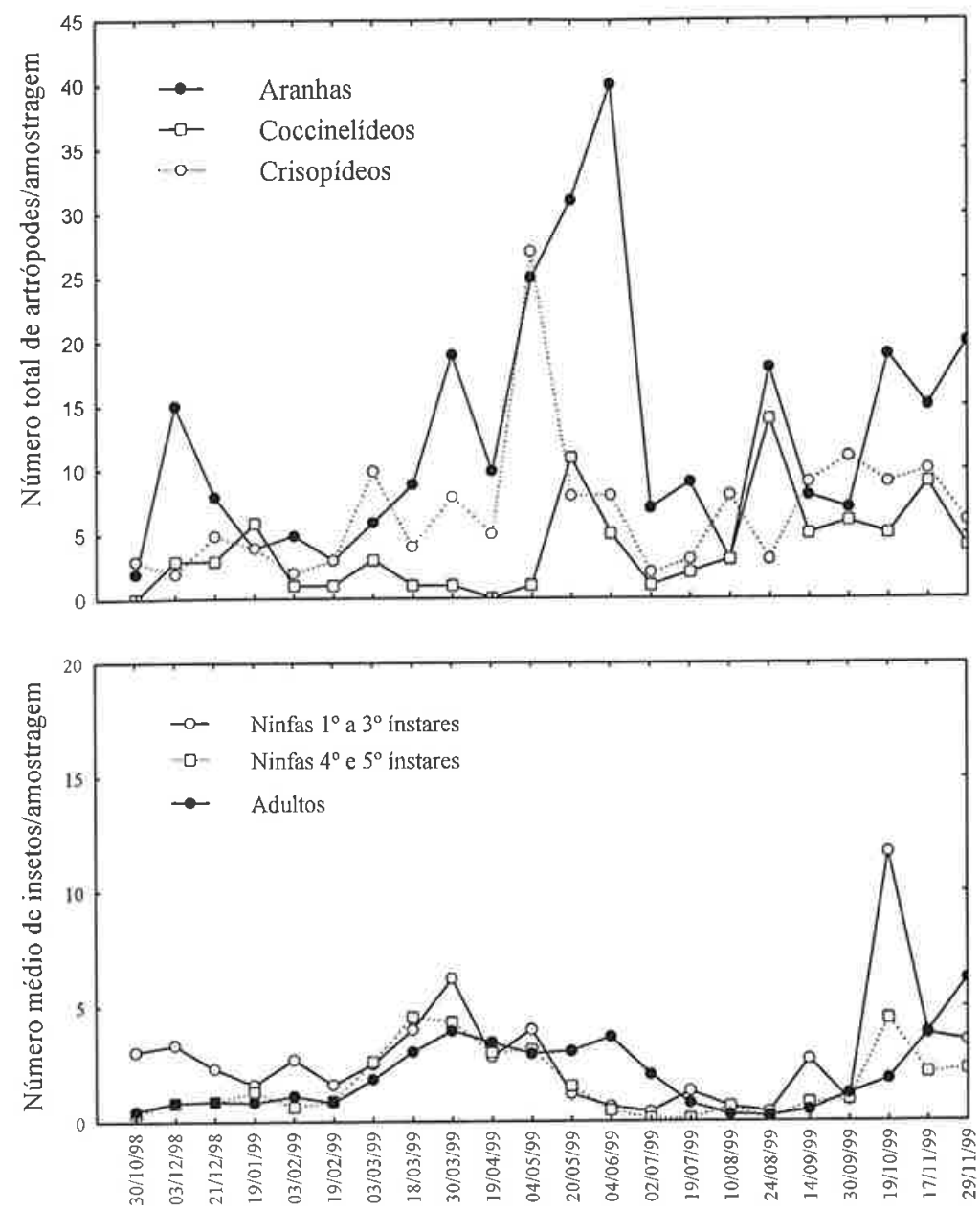

Datas de amostragem

Figura 1. Flutuação populacional de Leptopharsa heveae e de artrópodes predadores em seringueira. Pindorama, SP - 1998/99. 
Tabela 1. Coeficientes de correlação (r) obtidos entre o número médio de Leptopharsa heveae e o total de artrópodes predadores. Pindorama, SP 1998/99.

\begin{tabular}{lcccc}
\hline & \multicolumn{4}{c}{ L. heveae } \\
\cline { 2 - 5 } Família/ Espécie & $\mathrm{Np}$ & $\mathrm{Ng}$ & $\mathrm{N}$ & $\mathrm{A}$ \\
\hline Anyphaenidae & 0,28 & 0,14 & 0,24 & 0,42 \\
Dictynidae & 0,20 & 0,18 & 0,20 & $0,54^{*}$ \\
Salticidae & $-0,13$ & 0,05 & $-0,07$ & 0,33 \\
Araneidae & $-0,00$ & 0,18 & 0,07 & 0,33 \\
Total & 0,15 & 0,23 & 0,19 & $0,61^{*}$ \\
\hline Coccinellidae & & & & \\
Scymnus sp. & $-0,10$ & $-0,08$ & $-0,10$ & $-0,02$ \\
Diomus sp. & $-0,03$ & $-0,05$ & $-0,04$ & 0,20 \\
C. sanguinea & $-0,20$ & $-0,26$ & $-0,24$ & $-0,25$ \\
Total & $-0,22$ & $-0,25$ & $-0,25$ & $-0,12$ \\
\hline Chrysopidae & 0,20 & 0,37 & 0,28 & 0,27 \\
\hline
\end{tabular}

Significativo a 5\%.

$\mathrm{Np}, \mathrm{Ng}, \mathrm{N}, \mathrm{A}=$ ninfas de $1^{\circ}$ a $3^{\circ}$ instares, ninfas de $4^{\circ}$ e $5^{\circ}$ ínstares, total de ninfas e adultos, respectivamente. 